\title{
Satellite Image Resolution Enhancement Technique Using DWT and IWT
}

\author{
E. Sagar Kumar \\ Dept of ECE (DECS), \\ Vardhaman College of Engineering,
}

\author{
MR. T. Ramakrishnaiah \\ Assistant Professor (Sr.Grade), \\ Vardhaman College of Engineering,
}

\begin{abstract}
Now a days satellite images are widely used In many applications such as astronomy and geographical information systems and geosciences studies .In this paper, We propose a new satellite image resolution enhancement technique which generates sharper high resolution image .Based on the high frequency sub-bands obtained from the dwt and iwt. We are not considering the LL sub-band here. In this resolution-enhancement technique using interpolated DWT and IWT high-frequency sub band images and the input low-resolution image. Inverse DWT (IDWT) has been applied to combine all these images to generate the final resolution-enhanced image. The proposed technique has been tested on satellite bench mark images. The quantitative (peak signal to noise ratio and mean square error) and visual results show the superiority of the proposed technique over the conventional method and standard image enhancement technique WZP.
\end{abstract}

Keywords: DWT; Interpolation;IWT; Resolution; WZP;

\section{I.INTRODUCTION}

Resolution has been frequently referred as an important aspect of an image. Images are being processed in order to obtain more enhanced resolution. One of the commonly used techniques for image resolution enhancement is Interpolation. Interpolation has been widely used in many image processing applications such as facial reconstruction, multiple description coding, and super resolution. There are three well known interpolation techniques, namely nearest neighbor interpolation, bilinear interpolation, and bi-cubic interpolation. The results are decomposed along the columns. This operation results in four decomposed sub band images referred to low(LL), low-high (LH), high-low (HL), and high-high (HH).The frequency components of those sub bands cover the full frequency spectrum of the original image. Image resolution enhancement using wavelets is a relatively new subject and recently many new algorithms have been proposed. Their estimation was carried out by investigating the evolution of wavelet transform extreme among the same type of sub bands. Edges identified by an edge detection algorithm in lower frequency sub bands were used to prepare a model for estimating edges in higher frequency sub bands and only the coefficients with significant values were estimated as the evolution of the wavelet coefficients. In many researches, hidden Markov has been also implemented in order to estimate the coefficients. The proposed technique has been compared with conventional and state-of-art image resolution enhancement techniques. The conventional techniques used are the following interpolation techniques: bilinear interpolation and bi-cubic interpolation, wavelet zero padding (WZP),DWT based super resolution (DWT SR)[1].According to the quantitative and qualitative experimental results, the proposed technique over performs the aforementioned conventional and state-of-art techniques for image resolution enhancement. 


\section{International Journal of Computer Applications Technology and Research Volume 4- Issue 1, 70 - 76, 2015, ISSN:- 2319-8656}

\section{INTEGER WAVELET TRANSFORM (IWT)}

Integer to integer wavelet transforms (IWT)[2] maps an integer data set into another integer data set. This transform is perfectly invertible and yield exactly the original data set. A one dimensional discrete wavelet transform is a repeated filter bank algorithm. The reconstruction involves a convolution with the syntheses filters and the results of these convolutions are added. In two dimensions, we first apply one step of the one dimensional transform to all rows. Then, we repeat the same for all columns. In the next step, we proceed with the coefficients that result from a convolution in both directions. As shown in figure 1, these steps result in four classes of coefficients: the $(\mathrm{HH})$ coefficients represent diagonal features of the image, whereas (HL and LH) reflect vertical and horizontal information. At the coarsest level, we also keep low pass coefficients (LL). We can do the same decomposition on the LL quadrant up to $\log 2(\min$ (height, width)). Since the discrete wavelet transform allows independent processing of the resulting components without significant perceptible interaction To make the process of imperceptible embedding more effective. However, the used wavelet filters have floating point coefficients. Thus, when the input data consist of sequences of integers (as in the case for images), the resulting filtered outputs no longer consist of integers, which doesn't allow perfect reconstruction of the original image. However, with the introduction of Wavelet transforms that map integers to integers we are able to characterize the output completely with integers

\section{CONVENTIONAL METHOD}

As it was mentioned before, resolution is an important feature in satellite imaging, which makes the resolution enhancement of such images to be of vital importance as increasing the resolution of these images will directly affect the performance of the system using these images as input. The main loss of an image after being resolution enhanced by applying interpolation is on its high-frequency components, which is due to the smoothing caused by interpolation. Hence, in order to increase the quality of the enhanced image, preserving the edges is essential. In this paper, DWT has been employed in order to preserve the high-frequency components of the image. DWT separates the image into different sub band images, namely, LL, LH, HL, and HH. A high-frequency sub band contains the high frequency component of the image. The interpolation can be applied to these four sub band images. In the wavelet domain, the lowresolution image is obtained by low-pass filtering of the high-resolution image. The low resolution image (LL sub band), without quantization (i.e., with double-precision pixel values) is used as the input for the proposed resolution enhancement process. In other words, low frequency sub band images are the low resolution of the original image. Therefore, instead of using low-frequency sub band images, which contains less information than the original input image, we are using this input image through the interpolation process. Hence, the input low-resolution image is interpolated with the half of the interpolation factor, $\alpha / 2$, used to interpolate the high-frequency sub-bands, as shown inFig. 2 In order to preserve more edge information, i.e., obtaining a sharper enhanced image, we have proposed an intermediate stage in high frequency sub band interpolation process.

As shown in Fig.1, the low-resolution input satellite image and the interpolated LL image with factor 2 are highly correlated. The difference between the LL sub band image and the lowresolution input image are in their high-frequency components. Hence, this difference image can be use in the intermediate process to correct the estimated high-frequency components. This estimation is performed by interpolating the highfrequency sub bands by factor 2 and then including the difference image (which is highfrequency components on low-resolution input image) into the estimated high-frequency images, followed by another interpolation with factor $\alpha / 2$ in order to reach the required size for IDWT process. 


\section{International Journal of Computer Applications Technology and Research Volume 4- Issue 1, 70 - 76, 2015, ISSN:- 2319-8656}

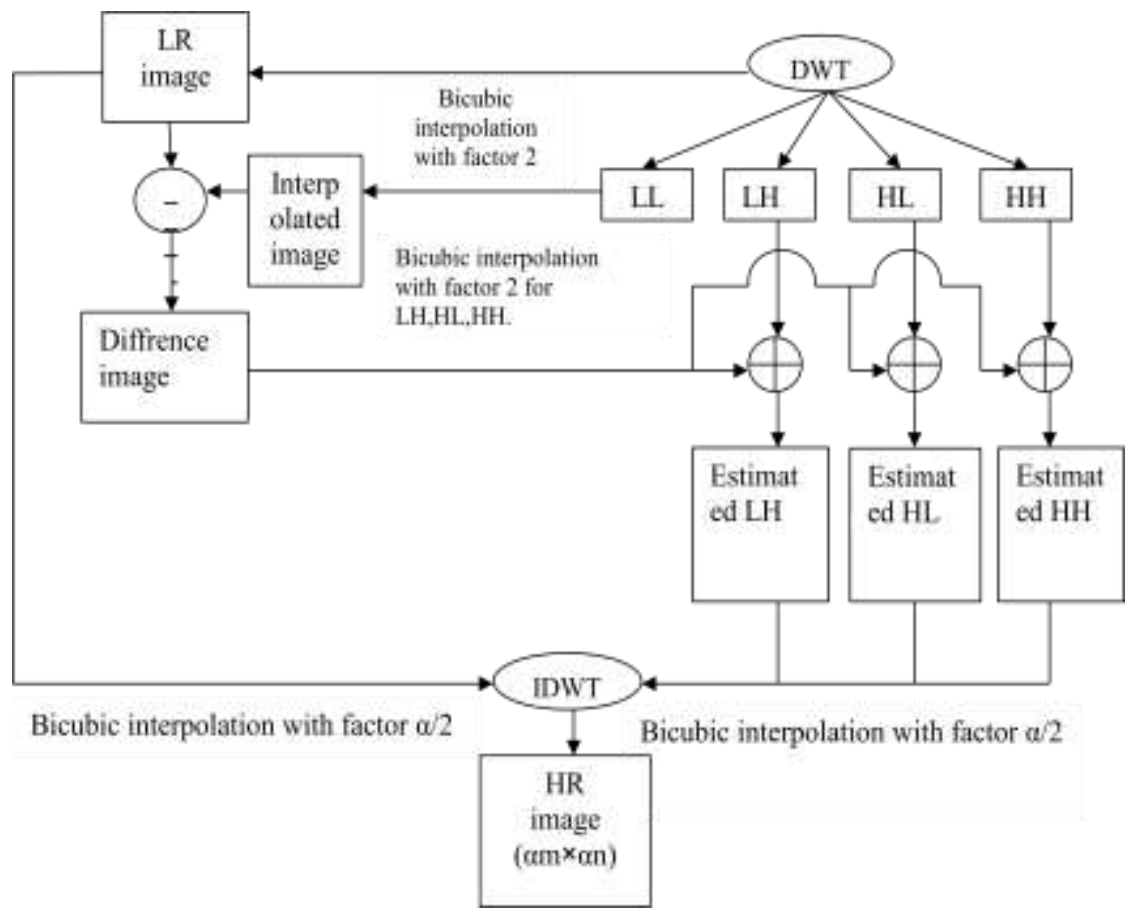

Fig.1 Conventional enhancement block diagram

\section{IV.PROPOSED METHOD}

A high-frequency sub band contains the high frequency component of the image. In other words, low frequency sub band images are the low resolution of the original image.Therefore, instead of using lowfrequency sub band images, which contains less information than the original input image. we are using this input image through the interpolation process. IWT also separates the image into different sub band images, namely, LL, LH, HL, and HH. A high-frequency sub band contains the high frequency component of the image. Hence, the input low-resolution image is interpolated with the half of the interpolation factor $\alpha / 2$, used to interpolate the high frequency sub bands, as shown in Fig.2.In order to preserve more edge information, i.e., obtaining a sharper enhanced image we have proposed an intermediate stage in high frequency sub band interpolation process. The DWT's LH sub band and IWT's LH sub band are added, and then interpolated by factor 2 to form estimated LH. The same procedure is repeated for $\mathrm{HL}$ and $\mathrm{HH}$ to form estimated $\mathrm{HL}$ and estimated $\mathrm{HH}$ respectively. In this process we are not considering LL sub bands of both DWT and IWT. In the place of LL we are taking input low resolution image. After estimation input low resolution image and high-frequency sub bands are interpolated by factor $\alpha / 2$ and to reach the required size for IDWT process. 


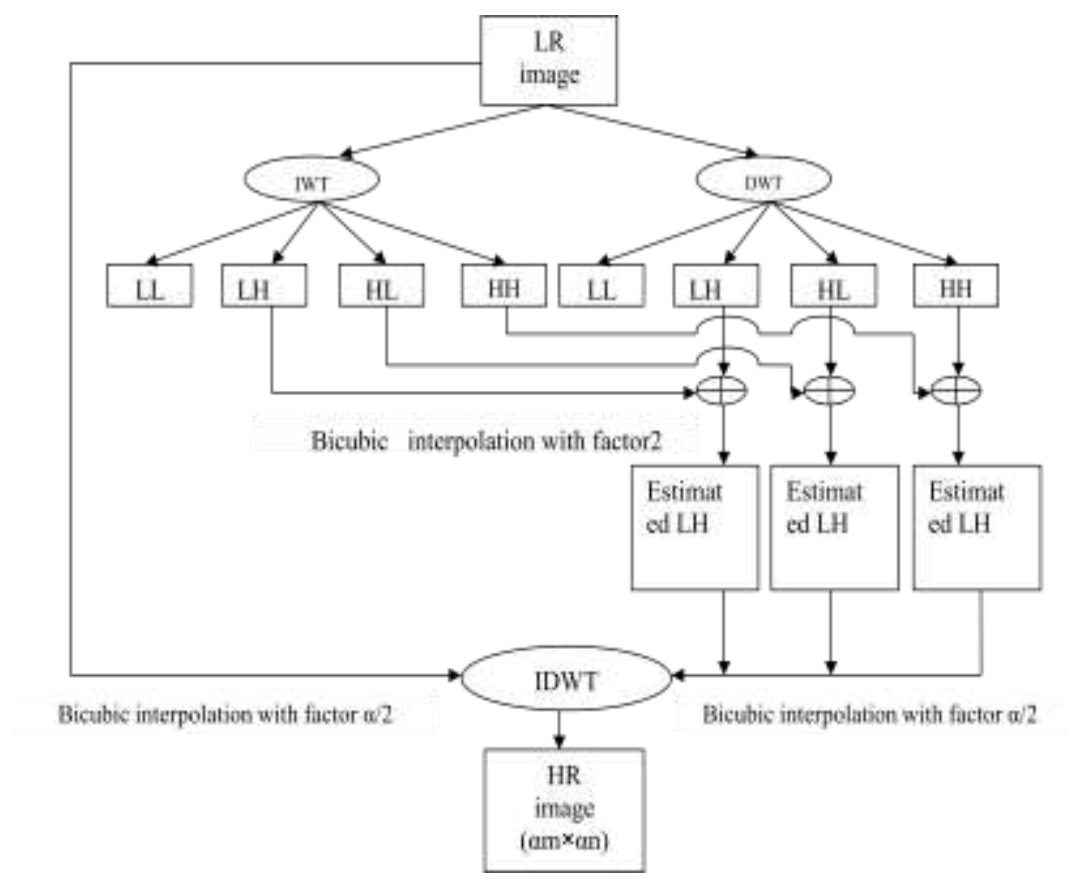

Fig.2 Proposed method Block Diagram

\section{V.CONCLUSION}

Satellite image enhancement is area of concern in the field of digital image processing. In literature many conventional works has been reported but fails to achieve desired enhancement results based on PSNR and MSE parameters based on superiority of the output images. In this paper a new enhancement technique has been reported based on the interpolation of the high frequency sub band images by DWT, IWT and the input low resolution image. The PSNR improvement of the proposed technique is upto $2.19 \mathrm{~dB}$ compared with conventional method.

\section{VI.ACKNOWLEDGMENT}

The authors would like to thank Dr. A.Temizel from Bilkent University for providing the output of the WZP resolution enhancement technique [14]. Moreover, the authors would like to acknowledge Prof. Dr. I. Selesnick from Polytechnic University for providing the DWT codes in MATLAB. Furthe rmore, the authors would like to thank Google Earth and Satellite Imaging Corporation for providing satellite images for research purposes. 


\section{VII.SIMULATION RESULTS}

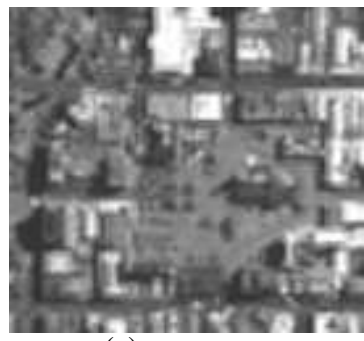

(a)

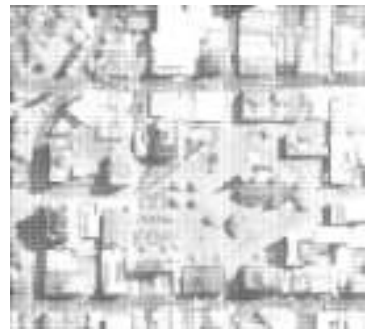

(b)

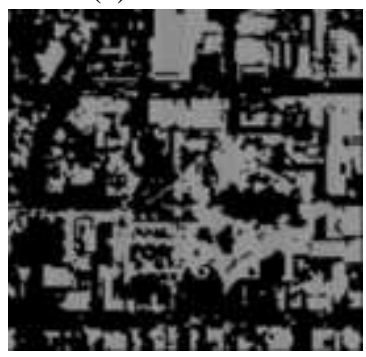

(c)

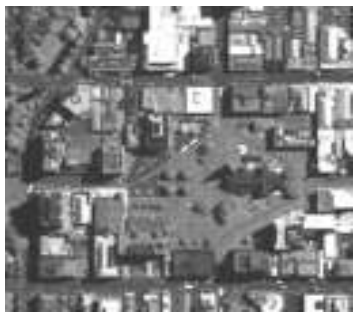

(d)

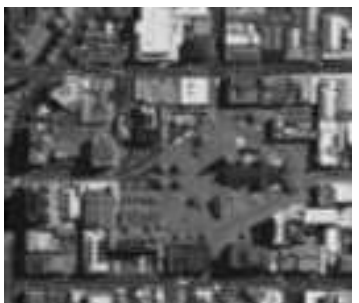

(e)

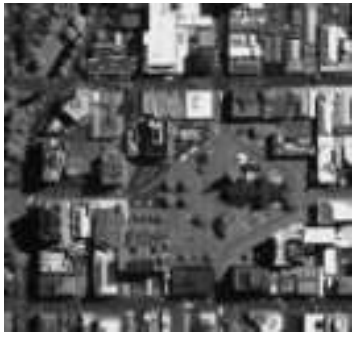

(f)

(a) low resolution image, (b) Bilinear image (c) Bicubic image (d) Super resolution image using WZP(e) DWT based image resolution enhancement output, (f) proposed method output image.

In the above images the proposed method output image superiority is better than the input image and all conventional methods output images.
Hence it is called best enhancement method for low resolution satellite images.

www.ijcat.com 
International Journal of Computer Applications Technology and Research Volume 4- Issue 1, 70 - 76, 2015, ISSN:- 2319-8656

\begin{tabular}{|l|l|l|}
\hline \multicolumn{1}{|c|}{ Enhancement Method } & \multicolumn{1}{c|}{ PSNR } & \multicolumn{1}{c|}{ MSE } \\
\hline Bi-Linear & 25.7841 & 0.4247 \\
\hline Bi-cubic & 29.7570 & 0.2697 \\
\hline WZP & 35.6436 & 0.0695 \\
\hline DWT & 35.6700 & 0.0690 \\
\hline Proposed & 37.6488 & 0.0438 \\
\hline
\end{tabular}

TABULAR COLUMN: PSNR and MSE Results for resolution enhancement from $128 \times 128$ to $512 \times 512(\alpha=4)$ for the proposed technique compared with conventional resolution enhancement techniques.

\section{VIII.REFERENCES}

[1]Hasan Demirel and Gholamreza Anbarjafari, "Discrete Wavelet Transform-Based Satellite Image Resolution Enhancement", IEEE transactions on geosciences and remote sensing, June 2013.

[2]Integer Wavelet transform using the lifting scheme.Geert uyterhoeven,Dirk Roose, adhemar, Bultheel,Department of computer science.

[3] H. Demirel, G. Anbarjafari, and S. Izadpanahi, "Improved motion-based localized super resolution technique using discrete wavelet transform for low resolution video enhancement," in Proc. 17th EUSIPCO, Edinburgh, U.K., Aug. 2009, pp. 1097-1101.

[4] L. Yi-bo, X. Hong, and Z. Sen-yue, "The wrinkle generation method for facial reconstruction based on extraction of partition wrinkle line features and fractal interpolation," in Proc. 4th ICIG, Aug. 22-24, 2007, pp. 933937.

[5] Y. Rener, J. Wei, and C. Ken, "Downsample-based multiple description

coding and post-processing of decoding," in Proc. 27th CCC, Jul. 16-18, 2008, pp. 253-256.

[6] C. B. Atkins, C. A. Bouman, and J. P. Allebach, "Optimal image scaling using pixel classification," in Proc. ICIP, Oct. 7-10, 2001, vol. 3, pp. 864867.

[7] Y. Piao, L. Shin, and H. W. Park, "Image resolution enhancement using inter-subband correlation in wavelet domain," in Proc. IEEE ICIP, 2007,

[8] G. Anbarjafari and H. Demirel, "Image super resolution based on interpolation

of wavelet domain high frequency subbands and the spatial

domain input image," ETRI J., vol. 32, no. 3, pp. 390-394,

Jun. 2010.

[9] W. K. Carey, D. B. Chuang, and S. S. Hemami, "Regularity-preserving image interpolation," IEEE Trans. Image Process., vol. 8, no. 9, pp. 12951297, Sep. 1999.

[10] X. Li and M. T. Orchard, "New edge-directed interpolation," IEEE Trans.

Image Process., vol. 10, no. 10, pp. 1521-1527, Oct. 2001.

[11] K. Kinebuchi, D. D. Muresan, and T.W. Parks, "Image interpolation using wavelet based hidden Markov trees," in Proc. IEEE ICASSP, 2001, vol. 3, pp. 7-11.

[12] M. S. Crouse, R. D. Nowak, and R. G. Baraniuk, "Wavelet-based statistical 
signal processing using hidden Markov models," IEEE Trans. Signal

Process., vol. 46, no. 4, pp. 886-902, Apr. 1998.

[13] S. Zhao, H. Han, and S. Peng, "Wavelet domain HMT-based image super

resolution," in Proc. IEEE ICIP, Sep. 2003, vol. 2, pp. 933-936.

[14] A. Temizel and T. Vlachos, "Image resolution upscaling in the wavelet

domain using directional cycle spinning," J. Electron. Imaging, vol. 14, no. 4, p. 040501, 2005.

[15] A. Gambardella andM.Migliaccio, "On the superresolution of microwave scanning radiometer measurements," IEEE Geosci. Remote Sens. Lett.

vol. 5, no. 4, pp. 796-800, Oct. 2008.

[16] V. A. Tolpekin and A. Stein, "Quantification of the effects of landcoverclass

spectral separability on the accuracy of Markov-random-field-based superresolution mapping," IEEE Trans. Geosci. Remote Sens., vol. 47, no. 9, pp. 3283-3297, Sep. 2009. [17] A. Temizel and T. Vlachos, "Wavelet domain image resolution enhancement

using cycle-spinning," Electron. Lett., vol. 41, no. 3, pp. 119-121,

Feb. 3, 2005.

[18] L. A. Ray and R. R. Adhami, "Dual tree discrete wavelet transform

with application to image fusion," in Proc. 38th Southeastern Symp. Syst.

Theory, Mar. 5-7, 2006, pp. 430-433.

[19] A. Temizel, "Image resolution enhancement using wavelet domain hidden

Markov tree and coefficient sign estimation," in Proc. ICIP, 2007, vol. 5, pp. V-381-V-384.

[20] R. C. Gonzalez and R. E. Woods, Digital Image Processing. Englewood

Cliffs, NJ: Prentice-Hall, 2007. 